\title{
The critical condition of a sewage network
}

\author{
Andrzej Raganowicz ${ }^{1, *}$, Józef Dziopak ${ }^{2}$ \\ ${ }^{1}$ Zweckverband zur Abwasserbeseitigung im Hachinger Tal, 82024 Taufkirchen, Germany \\ ${ }^{2}$ Rzeszów University of Technology, 35-959 Rzeszów, Poland
}

\begin{abstract}
The paper presents the methodology formulated for determining the critical technical state of operating sewer pipes which is based on statistical-stochastic modeling. It describes the theoretical boundary between the area comprised of pipes that do not show any signs of damage or require only maintenance measures, and the area comprised of pipes that require renovation. The study includes concrete collectors with dimensions of DN 600/1100-900/1350 mm, stoneware pipes (DN 200-400 mm), and stoneware connections (DN 100-200 mm). The modeling phase was carried out using the Weibull distribution in combination with Monte-Carlo mathematical simulations.
\end{abstract}

\section{Introduction}

The object of the research is the sewage infrastructure that operates in the catchment of the Hachinger Bach stream. The operator of the sewage infrastructure is the Target Association called Zweckverband zur Abwasserbeseitigung im Hachinger Tal. This linear sewage object discharges domestic wastewater from three municipalities in Bavaria - Oberhaching, Taufkirchen, and Unterchahing - located in the south-east part of Munich.

The material for the sewage network with diameters of DN $200-400 \mathrm{~mm}$ is stoneware. The transit of wastewater takes place in two concrete collectors of DN 600/1100-900/1350 $\mathrm{mm}$. The total length of the stoneware and concrete pipes is $150 \mathrm{~km}$. Another important component of this network are stoneware connections of DN 100-200 mm joining the public canals with revision wells of a total length of $95 \mathrm{~km}$.

In the framework of statistical-stochastic studies, the critical technical-operational state of the stoneware and concrete pipes of a sewage network and stoneware connections' condition were established. It sets a border between the area comprised of pipes without any damage or which require only maintenance measures, and the area comprised of pipes which only requiring renovation.

The paper presents a series of studies which are a simplified version of a network technical state forecasting. The statistical approximation of the state is based on Weibull distribution, and to estimate its parameters the Gumbel method was used. However, in the phase of stochastic modeling for the estimation of Weibull parameters the Gumbel method in combination with the Monte-Carlo method was used.

\footnotetext{
*Corresponding author: a-raganowicz@t-online.de
} 


\section{Phase of statistical research}

The basic idea of statistical and stochastic modeling was the thesis that the technical condition of sewage pipes depends on their age. The basis for the empirical data was three random samples generated from the examination of concrete, stoneware pipes and stoneware connections. Concrete and stoneware pipes are functioning in the municipality of Unterhaching and are located above the groundwater table. An optical inspection of these pipes was carried out in 2000. The first set of random sample is 125 sections of concrete pipes, and the second - up to 1162 sections of stoneware pipes. The technical state of the concrete and stoneware pipes was classified using a five-point scale: from fourth class being the best, to zero class being the worst, according to the German guideline ATV-M 149 [1]. From the best three classes of technical condition (ZK4, ZK3 and ZK2) additional random samples were created that formed the basis for the statistical modeling of the critical state of concrete and stoneware pipes. The number of reliable random samples was 84 sections of concrete pipes and 1122 sections of stoneware pipes. Both random samples represented the so-called third class of renovation priority (KO 5-3).

The third random sample was stoneware connections composed of 673 facilities operating in the municipality of Oberhaching which are located above the groundwater table. An optical inspection of these objects was implemented in 2010. Classification of their state was carried out on a three-point scale, from the third class being the best (III) to the first class being the worst (I) according to DIN 1986-30 [2]. From the connections of the two best classes of technical condition (III + II) a new random sample was created, which was considered reliable for statistical modeling and consisted of 509 connections. The general characteristics of the three reliable random samples representing concrete and stoneware pipes and stoneware connections are shown in Table 1. Each section of the examined network was located between two revision wells, and each connection was assigned with certain age which was related to the optical inspection period, and that was supported by technical documentation. In order to simplify the modeling process, age groups for the sections were introduced with five-year intervals, i.e. $5,10, \ldots \ldots ., 45$.

Table 1. General characteristics of random sampling.

\begin{tabular}{|c|c|c|c|c|c|}
\hline Sample & $\begin{array}{c}\text { Number of units } \\
\text { of class KO 5-1 }\end{array}$ & $\begin{array}{c}\text { Number of units } \\
\text { of class KO 5-3 }\end{array}$ & $\begin{array}{c}\text { Length } \\
(\mathbf{m})\end{array}$ & Material & $\begin{array}{c}\text { Diameter } \\
\text { (mm) }\end{array}$ \\
\hline Nr. 1 & 125 & 84 & 5572 & Concrete & $600 / 1100-900 / 1350$ \\
Nr. 2 & 1162 & 1122 & 36784 & Stoneware & $200-400$ \\
Nr. 3 & 673 & 509 & 828 & Stoneware & $100-200$ \\
\hline
\end{tabular}

The prepared base of empirical data in the form of three representative random samples 1,2 and 3 were subjected to a preliminary statistical analysis. For each random sample, the empirical density function $f^{*}(t)$ and distribution of probability of pipe critical technical state occurrence $F^{*}(t)$ was established. On the basis of empirical function analysis it was assumed that theoretical curves of transition from the state of maintenance to the state of renovation of the examined sewage pipe types will be constructed on the basis of twoparameter Weibull distribution.

In relation to the sewage net objects viability distribution function $F(t / T, b)$ is the probability that the technical viability of a particular section of sewer pipe is equal to $t$. The first parameter of Weibull distribution is the characteristic service life $T$, which determines the scale of the curve along the $x$ axis. Characteristic service life can be considered as an average value of the Weibull distribution and, therefore, in the case of an increase of this 
parameter, a shift of reliability in the direction of long technical life follows. The second parameter $b$ is a measure of distribution steepness and asymmetry.

The Weibull model has the following mathematical conditions [3]:

- density function

$$
f(t)=F(t) / d(t)
$$

- failure function

$$
F(t)=1-\exp (-t / T)^{b}
$$

- reliability function

$$
R(t)=\exp (-t / T)^{b}
$$

where:

$t$ - statistical variable, (for example, the service life of the object in years),

$T$ - characteristic service life of distribution corresponding to the mean value of distribution (in years),

$b$ - parameter of distribution form.

In analyzing the given conditions, the construction and operation status of the network $t$ can be determined by setting the theoretical curve of probability of reliability $R(t)$ for each class. The concept of failure in the case of sewage networks should not be taken literally, but as a transition of the group of network segments from one class to the next, the weak class of technical condition in a very short period of time. Thus, the aging of the sewage infrastructure can be interpreted as a process of permanent transition from one state to the next, worse state. Analysis of the theoretical probability function of reliability allows determining a number of important operating parameters, including: (1) the mean time of sections staying in a definite class of condition, (2) the percentage rate of aging segments, and (3) the number of segments that require renovation. On the basis of data obtained from the statistical forecasting of the network technical state, the necessary range of renovation investment can be carefully planned in order to keep it in full availability for the planned time period.

The present research allows us to reduce full network technical condition forecasting to one critical key curve of transition from conservation state to renovation state considering its importance in terms of application. In order to determine the critical curves of the technical condition of the concrete and stoneware pipes and connections, an estimation of shape parameter and characteristic technical viability was performed by the Gumbel method using the dependence [3]:

$$
\begin{gathered}
\hat{b}=0,577 / S_{\log } \\
\hat{T}=10^{\left(\sum_{1}^{n}\left(\log \left|t_{i}\right|\right)\right) / n+0,2507 / \hat{b}}
\end{gathered}
$$

where:

$t_{i}$ - age of sewer pipe, years,

$S_{\log }$ - standard logarithmic deviation

The values of the parameters determined by the Gumbel method while considering the three groups of sewer pipes are shown in Table 2. An analysis of thedata in Table 2 shows that concrete and stoneware pipes demonstrate values similar to the characteristic value of technical viability equal to $29.426-29.792$ years. In contrast, connections take a slightly lower value of the parameter -27.728 years. 
In the context of shape parameter estimation theoretical transition curves for stoneware pipes and connections similar values of inclination of 2.089-2.122 were obtained. For concrete pipes the critical inclination of the curve was much greater as it reached the value of 2.867 .

Based on the defined parameters of Weibull distribution, critical curves of technicaloperational state can be constructed for concrete and stoneware pipes and connections. However, in order to obtain more accurate results for sewer pipes reliability tests, an attempt to determine Weibull distribution parameters using the Monte-Carlo method was taken, and this approach provided a new perspective of the problem of examination and an evaluation of the network infrastructure technical state.

Table 2. Parameters of Weibull distribution according to Gumbel method.

\begin{tabular}{|c|c|c|c|}
\hline Parameter & $\begin{array}{c}\text { Sample } \\
\text { Nr. 1 }\end{array}$ & $\begin{array}{c}\text { Sample } \\
\text { Nr. 2 }\end{array}$ & $\begin{array}{c}\text { Sample } \\
\text { Nr. 3 }\end{array}$ \\
\hline$\hat{b}$ & 2.867 & 2.122 & 2.089 \\
\hline$\hat{T}$ (a) & 29.426 & 29.792 & 27.728 \\
\hline
\end{tabular}

\section{Estimation of Weibull parameters using the Monte-Carlo method}

The Monte-Carlo method refers to a wide group of numerical methods in which random numbers are used for the approximation of solutions and/or simulation of various processes. It is one of the few methods that allow obtaining accurate results within a reasonable computation time.

The stochastic Weibull parameter estimation is based on the inversion method belonging to the family of Monte-Carlo methods. The basis of this calculation procedure is based on the assumption that the inverse function $F^{-1}$ is approximately equal to the distribution function $F$ and on the fact that distribution function values are in the range of $F$ $: R \rightarrow(0,1)$. Appointing an age to sewer pipes on the basis of the inverse function formula, and putting the random numbers with uniform distribution from the interval $(0,1)$ in place of the inverse function a ready algorithm is obtained which can be used to perform any number of mathematical simulations for sewer pipes' age in accordance with formula (6) [4]:

$$
t_{i}^{k *}=\hat{T}\left(\ln \left(1 / 1-U_{i}^{k *}\right)\right) \frac{1}{\hat{b}}
$$

where:

$i=1,2, \ldots, \mathrm{n}$,

$k^{*}=1,2, \ldots ., N$,

$t_{i}^{k *}$ - simulated age of pipes (in years),

$\hat{b}$ - parameter of Weibull distribution shape determined by the Gumbel method,

$U_{i}^{k^{*}}$ - random numbers of uniform distribution of the interval $(0,1)$,

$\hat{T}$ - characteristic service life according to the Gumbel method (in years).

A necessary condition for many series of mathematical simulations is to have long chains of random numbers with unique sequences. Currently for these purposes, pseudo- random numbers generators are used. For the purposes of planned mathematical simulations, 
a pseudo-random numbers generator known as Multiplicative Linear Congruential Generator was used [5]. Its formula is described as follows:

$$
x_{i+1}=\left(\mathrm{a} x_{i}+\mathrm{b}\right) \bmod m
$$

where:

$-m=2^{32}$,

$-\mathrm{a}=69069$,

$-b=23,606,797$.

The constant $b$ often takes a value equal to zero. In contrast, the condition for the module $m$ must always be fulfilled, and so $0<a<m$. Module $m$ shows an area, in which random variables are sought. The length of the repeating numerical sequence in this case is $m$.

During this phase of research 500, 1000, 2500, 5000 and 10,000 mathematical simulations were carried out for each random sample. The values obtained in this way were added to formulas (4) and (5) in order to accurately determine Weibull distribution parameters. The results of Weibull parameters' estimation based on the Monte-Carlo method for concrete and stoneware pipes and stoneware connections are summarized in Table 3.

The conducted calculations show that as the number of simulations increase, the values of parameters $b$ and $T$ are becoming more stable. Weibull parameters determined on the basis of 10,000 simulations can be considered as meaningful, since the difference in values of $b$ at the 5000 and 10,000 simulations is marked only by a second decimal place. This observation can be applied to the research of all types of sewer pipelines.

Table 3. Parameters of Weibull distribution according to the Monte-Carlo method.

\begin{tabular}{|c|c|c|c|c|c|c|}
\hline \multirow{2}{*}{$\begin{array}{c}\text { Number } \\
\text { of mathematical } \\
\text { simulations }\end{array}$} & \begin{tabular}{c} 
Nr.1 \\
\cline { 2 - 7 }
\end{tabular} & $\boldsymbol{T}$ (a) & $\begin{array}{c}\text { Nr.2 } \\
\boldsymbol{b}\end{array}$ & $\boldsymbol{T}$ (a) & $\begin{array}{c}\text { Nr.3 } \\
\boldsymbol{b}\end{array}$ & $\boldsymbol{T}$ (a) \\
\hline Gumbel method & 2.867 & 29.426 & 2.122 & 29.792 & 2.089 & 27.728 \\
MMC(500) & 2.933 & 29.098 & - & - & - & - \\
MMC(1000) & 2.864 & 29.466 & - & - & 2.171 & 27.595 \\
MMC(2500) & 2.813 & 29.587 & 2.071 & 29.851 & 2.061 & 28.041 \\
MMC(5000) & 2.831 & 29.756 & 2.033 & 29.971 & 2.005 & 27.726 \\
MMC(10,000) & 2.852 & 29.069 & 2.069 & 30.239 & 2.051 & 27.801 \\
\hline
\end{tabular}

For example, analyzing the results of the research on concrete pipes, the maximum difference of the values of the $b$ parameter was 0.12 . In contrast, the difference between the results of the statistical and stochastic estimation reached a value equal to 0.015 . The results of the estimation of Weibull parameters for different types of pipes based on 10,000 mathematical simulations were considered as meaningful and the construction of the critical transition curves was started.

The results of the stochastic approximation of the critical state of concrete and stoneware pipes and connections in the form of a reliability function are shown in Fig. 1. These curves generally run a short distance from each other, indicating a close technical condition represented by the various types of pipes.

From the analysis of critical curves (Fig. 1) it can be concluded that the operation of the examined sewage facilities can be divided into two phases. The best condition for concrete pipes was shown within the first 30 years of operation, as they require the smallest amount of renovation. In contrast, a worse technical condition were present in stoneware pipes, and 
the worst were found in the stoneware connections. Such a structure of technical condition for the operation of a sewer system is in accordance with the commonly known operating experience. In order to generalize this thesis, it can be stated that sewer pipes operating in apublic area (main collectors) exhibit a significantly better technical condition than sewer connections. This phenomenon is confirmed by the theory of damage.

A well-known operation experience shows that the number of defects in a public area is ca. 50 units/1000 m, and in the area of connection it is twice as large and is ca. 100 units/1000 $\mathrm{m}$ (ratio $2: 1$ ). These data were confirmed by research conducted by the author in the Hachinger Bach stream catchment [6]. The fixed number of damages to the main collectors was 66 units/1000 m, and for connections - 137 units/1000 m (ratio $2.07: 1$ ).

After 30 years of operation, the structure of the technical condition of the examined sewage infrastructure significantly changed. As a result of the concrete pipes' curve inflection, which takes place after 30 years of operation, its inclination towards shorter technical viability increases. The consequence of such a course of the curve is the poor condition of concrete pipes. From this moment, the better technical condition is demonstrated by connections, and the best by stoneware pipes. Noteworthy is the fact that the curves for stoneware pipes and connections are characterized by a smaller inclination without an inflection point.

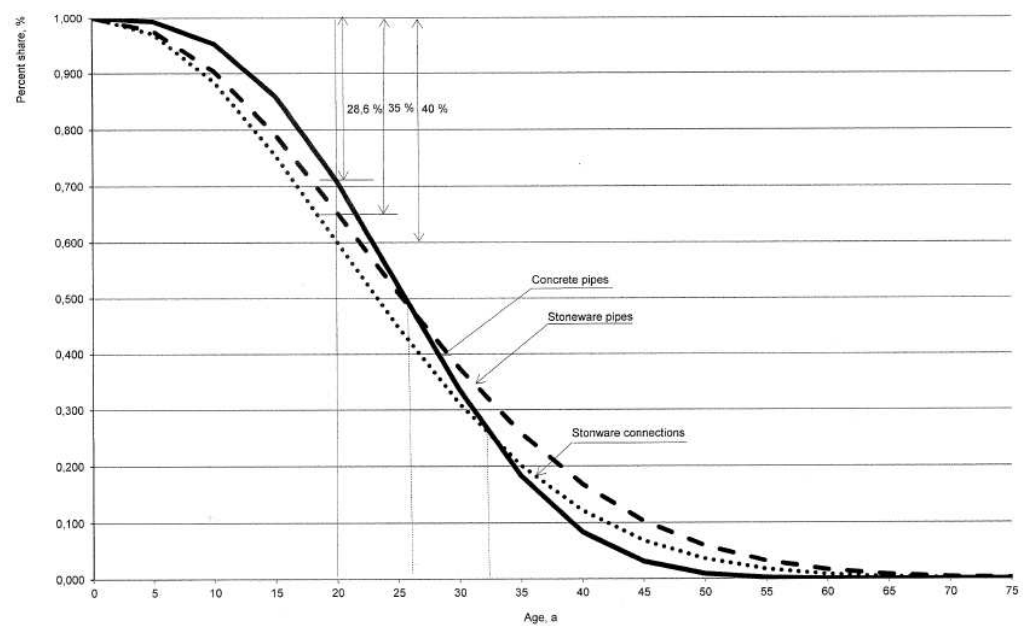

Fig. 1. Critical curves sequence for the technical and operational condition of concrete pipes, stoneware pipes and connections according to the Monte-Carlo method.

During their entire service life, stoneware pipes demonstrate a better technical condition than connections because their curve runs above the curve for connections. In the second phase of exploitation the connections are in a better technical state than the concrete pipes that should be considered as an anomaly typical for the network operating in the Hachinger Bach stream catchment. The operational design and technology for the installation of concrete pipes can be an attempt to explain this unusual phenomenon.

These large-size sewage objects (DN 600/1100-900/1350 mm) are rigid constructions made from unreinforced B10 concrete, for which the expansion joints were not foreseen. Even the inspection chambers are made from concrete monolith. The concrete collectors were performed using "wet" technology. In the previous pit, the bottom portion of the canal was first concreted as a single section with a length of $1.0 \mathrm{~m}$ to $1.5 \mathrm{~m}$, and then the vault was made. As a result of such adopted technologies, two longitudinal stitches were created, working on the entire length of the canal and the radial end of each section. As a result of various types of loads, tension is generated in these rigid structures, which in the absence of dilatation can be compensated only in the existing operation joints. 
This, as a result, leads to the formation of longitudinal and radial cracks, creating leakage from the pipes. Radial cracks act as joints, i.e. joints connecting the individual pipe sections. Another important aspect of the construction technology in concrete pipes was insufficient concrete compaction within the longitudinal operation joints. The operation of these facilities for 30 years, despite the implementation of a number of maintenance measures caused further adverse changes in the zones of operation joints. Taking into account the technology and the poor quality of concrete from which the pipes are made, it is understandable that their technical state after 30 years of operation was slightly worse than the state of the stoneware connections. Particularly noteworthy is the fact that concrete collectors in the Hachinger Bach stream catchment have been operating for over 50 years.

The second parameter of Weibull distribution is the characteristic lifetime $T$ which is dependent on the calculation of the shape parameter $b$. This parameter for both the statistical and stochastic approximation for stoneware and concrete pipes reached values in the range of $T=29-30$ years. However, for stoneware connections the vitality characteristic was $T=28$ years.

According to the elaborated prognosis for the critical technical state, the longest lifespan of stoneware pipes is approximately 70 to 75 years the stoneware pipes; while stoneware connections have a slightly shorter lifespan (65 years); and finally the shortest lifespan is for concrete pipes (55 to 60 years).

\section{Conclusions}

The range of statistical and stochastic research included three types of sewer pipes concrete pipes, stoneware pipes and stoneware connections that are the part of the sewage network discharging domestic wastewater from the Hachinger Tal stream catchment. The purpose of statistical-stochastic modeling was to identify the critical technical and operational state of sewer pipes.

The modeling of the critical state was conducted in two phases - statistical and stochastic. The statistical research phase was based on two-parameter Weibull distribution and the Gumbel method; and in the stochastic phase a combination of the Gumbel method and Monte-Carlo mathematical simulations was used. The use of the Monte-Carlo method allowed for an increase in the analyzed random samples.

The conducted modeling studies showed that statistical-stochastic forecast of critical technical and operational state is an effective form of analysis for the aging process of sewage facilities. The material used for the pipes and their role in a sewage system were taken into account within the framework of forecasting. The results of these studies confirmed the well-known operational experience that main pipes (public) exhibit better technical condition than the connections.

The proposed stochastic approximation of the critical technical and operational state of sewer pipes have a universal character and can be used in daily operating practice in order to effectively prevent the destruction of the linear urban infrastructure, which may even result in a building catastrophe. The methodology of sewer pipes' critical technical condition estimation can also be used in other areas of water management, for example to predict the level of the groundwater table or water level in surface streams.

\section{References}

1. ATV-M 149, Condition classification and evaluation of drainage systems outside buildings (1999)

2. DIN 1986-30, Drainage systems on private ground-Part 30: Maintenance (2012) 
3. H. Wilker, Weibull statistics in practice, guideline for reliability assessment of technical components, Books on Demand GmbH, Norderstedt (2010)

4. C. Cottin, S. Döhler, Risk analysis-modeling, assessment and management of risks with practical examples, Springer Fachmedien, Wiesbaden (2013)

5. F. Leisch, Computer intensive methoden, LMU München, (2011)

6. A. Raganowicz, The technical state of connections, AQUA\&GAS 6 (2013) 